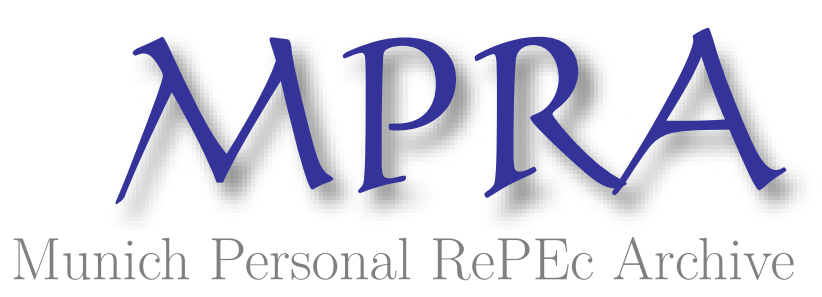

Parallel axiomatizations of weighted and multiweighted Shapley values, random order values, and the Harsanyi set

Besner, Manfred

15 March 2019

Online at https://mpra.ub.uni-muenchen.de/109331/

MPRA Paper No. 109331, posted 23 Aug 2021 13:48 UTC 


\title{
Parallel axiomatizations of weighted and multiweighted Shapley values, random order values, and the Harsanyi set
}

\author{
Manfred Besner*
}

April 2, 2019

\begin{abstract}
We present new axiomatic characterizations of five classes of TU-values, the classes of the weighted, positively weighted, and multiweighted Shapley values, random order values, and the Harsanyi set. The axiomatizations are given in parallel, i. e., they differ only in one axiom. In conjunction with marginality, a new property, called coalitional differential dependence, is the key that allows us to dispense with additivity. In addition, we propose new axiomatizations of the above five classes, in which, in part new, different versions of monotonicity, associated with the strong monotonicity in Young (1985), are decisive.
\end{abstract}

Keywords Cooperative game $\cdot$ Marginality $\cdot$ Strong monotonicity $\cdot$ Coalitional differential dependence $\cdot$ Weighted Shapley values · Harsanyi set

\section{Introduction}

Parallel axiomatizations of TU values or whole classes of TU values, i. e., the axiomatizations differ only in one axiom, are popular in cooperative game theory: on the one hand, they show similarities between TU values and, on the other hand, they work out important differences that are often only apparently small. Derks et al. (2000) offer a parallel axiomatization of the Harsanyi set (Hammer, 1977; Vasil'ev, 1978), the class of random order values (Weber, 1988), the class of weighted Shapley values (Shapley, 1953a; Kalai and Samet, 1987), and the Shapley value (Shapley, 1953b). All given axiomatizations apply additivity whereby the axiomatization of the weighted Shapley values requires five axioms instead of four in the other presented axiomatizations. We refer to this parallel axiomatization in the main part, replacing the five axiom axiomatization of the weighted Shapley values with two elegant new ones from Casajus (2019), using only four axioms, one for the weighted Shapley values and one for the subset of positively weighted Shapley values (Shapley, 1953a).

In addition, we remove the axiomatization of the Shapley value and include an axiomatization of the multi-weighted Shapley values (Dragan, 1992). The multiweighted Shapley values extend the Harsanyi payoffs, which are the TU-values from the Harsanyi set. Here,

\footnotetext{
${ }^{*}$ M. Besner

Department of Geomatics, Computer Science and Mathematics,

HFT Stuttgart, University of Applied Sciences, Schellingstr. 24

D-70174 Stuttgart, Germany

E-mail: manfred.besner@hft-stuttgart.de
} 
the previous non-negative players' sharing weights can be real numbers for each associated coalition, provided they add up to a sum of one for each of these coalitions. Although the multi-weighted Shapley values do not seem realistic for many applications, they have good theoretical significance and round off our axiomatizations.

While some axiomatizations without additivity are known for the weighted Shapley values, it looks unsatisfactory for the other classes in this respect. One of the most remarkable axiomatizations of the Shapley value without additivity comes from Young (1985). Besides efficiency and symmetry, Young uses strong monotonicity. As the proof shows, the axiomatization, in reality, requires only marginality. The symmetry axiom is such strong that the monotonicity part in strong monotonicity is redundant in this axiomatization.

In our first main result, we axiomatize the above-mentioned classes of TU-values in such a way that different varieties of monotonicity are decisive. Besides efficiency, monotonicity, and mutual dependence, Nowak and Radzik (1995) used marginality to axiomatize the weighted Shapley values. In the first main result, we show that strong monotonicity can replace marginality and the additional monotonicity property in this axiomatization. In order to axiomatize the other TU-value classes, we introduce a new weakly and a new strictly strong monotonicity axiom. Van den Brink et al. (2013) propose weak monotonicity, also a weaker version of strong monotonicity, to axiomatize the egalitarian Shapley values (Joosten, 1996). We can interpret marginality as a static norm: as long as a player's marginal contributions do not change, her payoff should not change. Strong monotonicity implies that a player's payoff does not decrease if his marginal contributions do not decrease. In addition, weak monotonicity presupposes that the worth of the grand coalition will not decrease either. Our weakly strong monotonicity axiom is also a considerable weakening of strong monotonicity: As long as a player's marginal contributions do not change, with the possibility that at most the marginal contribution to one coalition may rise, her payoff should not fall. In contrast, strictly strong monotonicity means that a player's payoff does not decrease if his marginal contributions do not decrease, and increases in all cases if it is ensured that his marginal contribution to at least one coalition has actually increased.

As a new crucial property within our article, we introduce the coalitional differential dependence axiom, a relaxation of mutual dependence. Mutual dependence means that the payoff ratios of two players should be the same in two different games where both players are productive only in coalitions with both players. For coalitional differential dependence consider two different games where both players are productive only in coalitions that contain both players. Let us look at the associated games where both players bring the same amount of change to all supersets of a given coalition, compared to the old games. Then the ratios of payoff differentials of each old and new associated game should be the same for each player.

Our second main result, a corollary of our first main result and the referred parallel axiomatic characterization, is a completely parallel axiomatic characterization of all above mentioned TU-value classes without additivity.

The article is organized as follows. Some preliminaries are given in Section 2. Section 3 is the main part in which we introduce new axioms and present new axiomatizations, mostly in parallel. Section 4 concludes. The Appendix (Section 5) contains all the proofs, related lemmas, and demonstrates the logical independence of the axioms used for axiomatization. 


\section{Preliminaries}

A TU-game $(N, v)$ on a non-empty and finite player set $N$ is given by a coalition function $v: 2^{N} \rightarrow \mathbb{R}, v(\emptyset)=0$. We are only dealing with a fixed player set $N$ so the latter is usually omitted as an argument. Each $S \subseteq N$ is called a coalition, $v(S)$ is called the worth of coalition $S$ and $\Omega^{S}$ denotes the set of all non-empty subsets of $S$. The set of all TU-games is denoted by $\mathbb{V}$; the null game $\mathbf{0}$ is given by $\mathbf{0}(S)=0$ for all $S \subseteq N$. A TU-game is called monotonic if $v(R) \leq v(S)$ for all $R, S \subseteq N$ such that $R \subseteq S$. The dividends $\Delta_{v}(S)$ (Harsanyi, 1959) are defined inductively by

$$
\Delta_{v}(S):=\left\{\begin{array}{l}
v(S)-\sum_{R \subsetneq S} \Delta_{v}(R), \text { if } S \in \Omega^{N}, \text { and } \\
0, \text { if } S=\emptyset .
\end{array}\right.
$$

An unanimity game $u_{S} \in \mathbb{V}, S \in \Omega^{N}$, is defined for all $T \subseteq N$ by $u_{S}(T)=1$ if $S \subseteq T$ and $u_{S}(T)=0$ otherwise. $v$ is called totally positive (Vasil'ev, 1975) if $\Delta_{v}(S) \geq 0$ for all $S \subseteq N . S \subseteq N$ is called essential in $v$ if $\Delta_{v}(S) \neq 0$. For $i \in N$ and $S \subseteq N \backslash\{i\}$, the marginal contribution $M C_{i}^{v}(S)$ is given by $M C_{i}^{v}(S):=v(S \cup\{i\})-v(S) ; i \in N$ is called a null player in $v$ if $v(S \cup\{i\})=v(S)$ for all $S \subseteq N \backslash\{i\}$; players $i, j \in N, i \neq j$, are called (mutually) dependent (Nowak and Radzik, 1995) in $v$ if $v(S \cup\{i\})=v(S)=v(S \cup\{j\})$. It is well-known that we have for dependent players $i, j \in N$ in $v$,

$$
\Delta_{v}(S)=0 \text { for all } S \subseteq N \text { such that }|S \cap\{i, j\}|=1 .
$$

A TU-value $\varphi$ is an operator that assigns to any $v \in \mathbb{V}$ a payoff vector $\varphi(v) \in \mathbb{R}^{N}$. We define by $W^{N}:=\left\{f: N \rightarrow \mathbb{R}_{++}\right\}, \mathrm{w}_{i}:=\mathrm{w}(i)$ for all $\mathrm{w} \in W^{N}, i \in N$, the collection of all positive weight systems on $N$; a weight system on $N$ is a pair (w, $\sigma)$ consisting of a positive weight system $\mathrm{w} \in W^{N}$ together with an ordered partition $\sigma:=\left\{S_{1}, \ldots, S_{h}\right\}$ of $N$, the collection of all weight systems on $N$ is denoted by $\mathcal{W}^{N}$; the collection of all sharing systems $\lambda \in \Lambda^{N}$ on $N$ is defined by

$$
\Lambda^{N}:=\left\{\lambda=\left(\lambda_{S, i}\right)_{S \in \Omega^{N}, i \in S} \mid \sum_{i \in S} \lambda_{S, i}=1 \text { and } \lambda_{S, i} \geq 0 \text { for each } S \in \Omega^{N} \text { and all } i \in S\right\},
$$

and the collection $\Lambda^{+}{ }^{N}$ of all extended sharing systems $\lambda^{+} \in \Lambda^{+}{ }^{-}$is defined by

$$
\Lambda^{+}{ }^{N}:=\left\{\lambda^{+}=\left(\lambda_{S, i}^{+}\right)_{S \in \Omega^{N}, i \in S} \mid \sum_{i \in S} \lambda_{S, i}^{+}=1 \text { for all } S \in \Omega^{N} \text { and all } i \in S\right\} .
$$

The positively weighted Shapley values $S h^{\mathrm{w}}$ (Shapley, 1953a) are given by

$$
S h_{i}^{\mathrm{w}}(v):=\sum_{S \subseteq N, S \ni i} \frac{\mathrm{w}_{i}}{\sum_{j \in S} \mathrm{w}_{j}} \Delta_{v}(S) \text { for all } i \in N \text { and } \mathrm{w} \in W^{N} .
$$

In the specific case that all weights are equal, we get the Shapley value Sh (Shapley, 1953b), defined by

$$
S h_{i}(v):=\sum_{S \subseteq N, S \ni i} \frac{\Delta_{v}(S)}{|S|} \text { for all } i \in N
$$


For all $(\mathrm{w}, \sigma) \in \mathcal{W}^{N}$, the weighted Shapley values $S h^{(\mathrm{w}, \sigma)}$ (Kalai and Samet, 1987) are given by

$$
S h_{i}^{(\mathrm{w}, \sigma)}(v):=\sum_{T \subseteq N, T(\sigma) \ni i} \frac{\mathrm{w}_{i}}{\sum_{j \in T(\sigma)} \mathrm{w}_{j}} \Delta_{v}(T) \text { for all } i \in N
$$

where $T(\sigma):=T \cap S_{m}, m:=\max \left\{\ell \mid T \cap S_{\ell} \neq \emptyset, S_{\ell} \in \sigma\right\}$. Note, if $\sigma=\{N\}$, we have $S h^{(\mathrm{w}, \sigma)}=S h^{\mathrm{w}}$. We denote the set of all weighted Shapley values by $\mathcal{S}$ and the set of all positively weighted Shapley values by $\mathcal{P} \mathcal{S}$.

Independently of each other, Hammer (1977) and Vasil'ev (1978) established a set of TUvalues called Harsanyi set, also known as selectope, that we denote by $\mathcal{H}$. The payoffs are made by distributing the Harsanyi dividends via a sharing system. The TU-values $H^{\lambda}, \lambda \in \Lambda^{N}$, from this set, titled Harsanyi payoffs ${ }^{1}$, are defined by the following,

$$
H_{i}^{\lambda}(v):=\sum_{S \subseteq N, S \ni i} \lambda_{S, i} \cdot \Delta_{v}(S) \text {, for all } i \in N .
$$

If we renounce the non-negativity of the sharing weights, we obtain the TU-value class $\mathcal{M}$ of all multiweighted Shapley values $M^{\lambda_{-}^{+}}$(Dragan, 1992), defined by

$$
M_{i}^{\lambda_{-}^{+}}(v)=\Delta_{v}(\{i\})+\sum_{S \subseteq N, S \ni i} \lambda_{S, i}^{+} \cdot \Delta_{v}(S) \text { for all } i \in N \text { and for each } \lambda^{+} \in \Lambda^{+} N
$$

Let $\Pi$ be the set of all permutations $\pi: N \rightarrow N$, and let $\pi^{i}:=\{j \in N \mid \pi(j) \leq \pi(i)\}$ the coalition of all predecessors of player $i \in N$, including player $i$. A TU-value $\psi$ is called a random order value (Weber, 1988) if there is a probability distribution $p=\left(p_{\pi}\right)_{\pi \in \Pi}, \sum_{\pi \in \Pi} p_{\pi}=1$, such that $\psi$ can be represented by

$$
\psi_{i}(v)=\sum_{\pi \in \Pi} p_{\pi} \cdot\left[v\left(\pi^{i}\right)-v\left(\pi^{i} \backslash\{i\}\right)\right], \text { for all } v \in \mathbb{V} \text { and } i \in N .
$$

The set of all random order values is denoted by $\mathcal{R}$.

We refer to the following well-known axioms for TU-values $\varphi$.

Efficiency, E. For all $v \in \mathbb{V}$, we have $\sum_{i \in N} \varphi_{i}(v)=v(N)$.

Null game, $\mathbf{N G}^{2} \cdot \varphi_{i}(\mathbf{0})=0$ for all $i \in N$.

Null player, $\mathbf{N}$. For all $v \in \mathbb{V}$ and $i \in N$ such that $i$ is a null player in $v$, we have $\varphi_{i}(v)=0$.

Additivity, A. For all $v, w \in \mathbb{V}$, we have $\varphi(v)+\varphi(w)=\varphi(v+w)$.

Homogenity, Ho. For all $\alpha \in \mathbb{R}$ and $v \in \mathbb{V}$, we have $\varphi(\alpha v)=\alpha \varphi(v)$.

Positivity, Po(Vasil'ev, 1975). For all $v \in \mathbb{V}$ such that $v$ is totally positive, we have $\varphi_{i}(v) \geq$ 0 for all $i \in N$.

Monotonicity, Mo. For all $v \in \mathbb{V}$ such that $v$ is monotonic, we have $\varphi_{i}(v) \geq 0$ for all $i \in N$.

Marginality, $\mathbf{M}$ (Young, 1985). For all $v, w \in \mathbb{V}$ and $i \in N$ such that $M C_{i}^{v}(S)=M C_{i}^{w}(S)$ for all $S \subseteq N \backslash\{i\}$, we have $\varphi_{i}(v)=\varphi_{i}(w)$.

Strong monotonicity, SM. (Young, 1985). For all $v, w \in \mathbb{V}$ and $i \in N$ such that $M C_{i}^{v}(S) \geq$ $M C_{i}^{w}(S)$ for all $S \subseteq N \backslash\{i\}$, we have $\varphi_{i}(v) \geq \varphi_{i}(w)$.

\footnotetext{
${ }^{1}$ These TU-values are also known as Harsanyi payoff vectors or sharing values.

${ }^{2}$ This axiom is also called triviality.
} 
Mutual dependence, MD (Nowak and Radzik, 1995). For all $v, w \in \mathbb{V}$ and $i, j \in N$ such that $i$ and $j$ are dependent in $v$ and $w$, we have $\varphi_{i}(v) \varphi_{j}(w)=\varphi_{i}(w) \varphi_{j}(v)$.

Weak sign symmetry, WSS (Casajus, 2019). For all $i, j \in N$ such that $i$ and $j$ are dependent in $v$, we have $\operatorname{sign}\left(\varphi_{i}(v)\right)=\operatorname{sign}\left(\varphi_{j}(v)\right)$.

Superweak sign symmetry, SSS (Casajus, 2019). For all $i, j \in N$ such that $i$ and $j$ are dependent in $v$, we have that $\operatorname{sign}\left(\varphi_{i}(v)\right)>0$ implies $\operatorname{sign}\left(\varphi_{j}(v)\right) \geq 0$.

\section{Parallel axiomatizations}

The claim of this article is to present parallel axiomatizations of some classes of TU values, which means that we have related axiomatizations that differ only in one axiom. At first, we recall that all classes considered are in a proper subset relationship.

Remark 3.1. We have $\mathcal{P S} \subsetneq \mathcal{S} \subsetneq \mathcal{R} \subsetneq \mathcal{H} \subsetneq \mathcal{M}$.

The relationship $\mathcal{S} \subsetneq \mathcal{R} \subsetneq \mathcal{M}$ is shown in Dragan (1992), $\mathcal{R} \subsetneq \mathcal{H}$ is shown in Derks et al. (2000), $\mathcal{H} \subsetneq \mathcal{M}$ follows by (2) and (3), and $\mathcal{P} \mathcal{S} \subsetneq \mathcal{S}$ is obvious by definition. The following theorem summarizes well-known axiomatizations into a parallel characterization.

Theorem 3.2. A TU-value $\varphi$ satisfies $\boldsymbol{E}, \boldsymbol{N}, \boldsymbol{A}$, and

(a) Ho if and only if $\varphi \in \mathcal{M}$ (Dragan, 1992).

(b) Po if and only if $\varphi \in \mathcal{H}$ (Vasil'ev, 1981; Derks et al., 2000).

(c) Mo if and only if $\varphi \in \mathcal{R}$ (Weber, 1988).

(d) $\boldsymbol{S S S}$ if and only if $\varphi \in \mathcal{S}$ (Casajus, 2019).

(e) $\boldsymbol{W S S}$ if and only if $\varphi \in \mathcal{P S}$ (Casajus, 2019).

Aside from $\mathbf{E}$ and $\mathbf{N}$, all these axiomatizations use $\mathbf{A}$ as a central property. The question arises whether it is possible to have parallel characterizations without this property. For the weighted Shapley values, some axiomatizations without additivity are well-known. Nowak and Radzik (1995) used $\mathbf{E}, \mathbf{M}, \mathbf{M o}$, and $\mathbf{M D}$ to axiomatize $\mathcal{S}$ and, by replacing $\mathbf{M o}$ with a stronger version, $\mathcal{P} \mathcal{S}$. MD states that if two players are dependent in two games on the same player set, the ratio of their payoffs in these two games is equal if in one game the payoffs of both players are not zero. MD is a rather strong property, and it is easy to prove that it does not apply to the other classes of values considered in Theorem 3.2.

The following axiom for two different games on the same player set is related. For each of the two games, we have an additional game in which the players of a coalition $S$, to which the dependent players $i$ and $j$ belong, change their cooperation and bring the same amount of change into all the supersets of $S$. Then the ratios of the payoff difference of each old and associated new game are the same for both players as long as the payoff differences in a game are not zero.

Coalitional differential dependence, CDD. For all $\alpha, \beta \in \mathbb{R}, v, w \in \mathbb{V}, S \subseteq N, i, j \in S$ such that $i$ and $j$ are dependent in $v$ and $w$, we have

$$
\begin{aligned}
& {\left[\varphi_{i}\left(v+\alpha \cdot u_{S}\right)-\varphi_{i}(v)\right]\left[\varphi_{j}\left(w+\beta \cdot u_{S}\right)-\varphi_{j}(w)\right]} \\
& \quad=\left[\varphi_{j}\left(v+\alpha \cdot u_{S}\right)-\varphi_{j}(v)\right]\left[\varphi_{i}\left(w+\beta \cdot u_{S}\right)-\varphi_{i}(w)\right]
\end{aligned}
$$


Remark 3.3. MD implies CDD but not vice versa.

The axiomatizations in Nowak and Radzik (1995, Theorem 2.4 and Remark 2.3) of the classes of weighted and positively weighted Shapley values have a strong connection to the axiomatization of the Shapley value in Young (1985). Young introduced SM that has the considerable meaning that a player's payoff should not decrease if the player's marginal contributions to all coalitions that do not include her do not decrease. Young used only $\mathbf{M}$ to prove his axiomatization and, in the words of Young (1985), M "is a type of independence condition rather than a monotonicity condition. ...The reason for emphasizing the somewhat stronger notion of monotonicity is that it seems to be the most relevant to actual applications." The following axiom can shed additional light on the effects of marginality.

Coalitional strategic equivalence, CSE (Chun, 1989). For all $v, w \in \mathbb{V}$ such that for any $T \in \Omega^{N}, c \in \mathbb{R}$, and all $S \subseteq N$,

$$
v(S)=\left\{\begin{array}{l}
w(S)+c, \text { if } S \supseteq T, \\
w(S), \text { otherwise, }
\end{array}\right.
$$

we have $\varphi_{i}(v)=\varphi_{i}(w)$ for all $i \in N \backslash T$.

Chun (1989) introduced this axiom to axiomatize the Shapley value. He assumed that his axiom is weaker than Young's strong monotonicity. But Casajus and Huettner (2008) have shown that this is not the case.

Proposition 3.4. (Casajus and Huettner, 2008) M and CSE are equivalent.

Therefore, CSE stresses the static property of marginality. The following property is placed somewhere in the middle between $\mathbf{M}$ and $\mathbf{S M}$.

Weakly strong monotonicity, WSM. For all $v, w \in \mathbb{V}$ and $i \in N$ such that $M C_{i}^{v}(T) \geq$ $M C_{i}^{w}(T)$ for one $T \subseteq N \backslash\{i\}$, and $M C_{i}^{v}(S)=M C_{i}^{w}(S)$ for all $S \subseteq N \backslash\{i\}, S \neq T$, we have $\varphi_{i}(v) \geq \varphi_{i}(w)$.

As long as at most one player's marginal contribution to a single coalition increases and all other marginal contributions remain unchanged, the payoff to that player will not decrease. Here, too, another axiom can additionally clarify the meaning of the axiom just introduced.

Coalitional strategic monotonicity, CSM. For all $v, w \in \mathbb{V}$ such that for any $T \in \Omega^{N}, c \in \mathbb{R}_{++}$, and all $S \subseteq N$,

$$
v(S)=\left\{\begin{array}{l}
w(S)+c, \text { if } S \supseteq T, \\
w(S), \text { otherwise, }
\end{array}\right.
$$

we have $\varphi_{i}(v)=\varphi_{i}(w)$ for all $i \in N \backslash T$ and $\varphi_{j}(v) \geq \varphi_{j}(w)$ for all $j \in T$.

Proposition 3.5. WSM and $\boldsymbol{C S M}$ are equivalent.

CSM makes it clear that WSM meets $\mathbf{M}$ on the one hand and has a slightly weaker monotonicity property than SM on the other. In addition, we see that WSM (and therefore SM and the following property where it is obvious) actually consists of two properties. The next property represents a change of $\mathbf{S M}$ to the opposite side. 
Strictly strong monotonicity, SSM. For all $v, w \in \mathbb{V}$ and $i \in N$ such that $M C_{i}^{v}(S) \geq$ $M C_{i}^{w}(S)$ for all $S \subseteq N \backslash\{i\}$, we have $\varphi_{i}(v) \geq \varphi_{i}(w)$ and if additionally $M C_{i}^{v}(T)>M C_{i}^{w}(T)$ for at least one $T \subseteq N \backslash\{i\}$, we have $\varphi_{i}(v)>\varphi_{i}(w)$.

SSM thus corresponds to SM with the additional characteristic that if a player's marginal contribution to at least one coalition actually increases, this player also receives a higher payoff.

Remark 3.6. The following implication is strict: $\mathrm{SSM} \Rightarrow \mathrm{SM} \Rightarrow \mathrm{WSM}$.

Casajus (2019) poses the question whether the classes of weighted/positively weighted Shapley values can be characterized by $\mathbf{E}, \mathbf{S M} / \mathbf{M}$, and SSS/WSS. However, we must reject this assumption. Consider the following TU-value $\phi$, defined as follows for the player set $N:=$ $\{i, j\}$ :

$$
\phi_{i}(v):=\left\{\begin{array}{l}
v(\{i\})+\frac{1}{3} \Delta_{v}(\{i, j\}), \text { if } \Delta_{v}(\{i, j\})<0 \\
S h_{i}(v), \text { otherwise }
\end{array}\right.
$$

and

$$
\phi_{j}(v):=\left\{\begin{array}{l}
v(\{j\})+\frac{2}{3} \Delta_{v}(\{i, j\}), \text { if } \Delta_{v}(\{i, j\})<0 \\
S h_{j}(v) \text { otherwise. }
\end{array}\right.
$$

Obviously, $\phi$ satisfies E, M, SM, SSS, and WSS but $\phi$ is no weighted Shapley value, yes $\phi$ is not even a multiweighted Shapley value at all! The next theorem gives a parallel axiomatic characterization of $\mathcal{M}, \mathcal{H}$, and $\mathcal{R}$ without additivity. In order to axiomatize $\mathcal{S}$ and $\mathcal{P} \mathcal{S}$, we must strengthen CDD to MD additionally. The various monotonicity axioms just discussed are decisive.

Theorem 3.7. A TU-value $\varphi$ satisfies $\boldsymbol{E}$,

(a) $\boldsymbol{N G}, \boldsymbol{C D D}$, and $\boldsymbol{M}$ if and only if $\varphi \in \mathcal{M}$.

(b) $\boldsymbol{N G}, \boldsymbol{C D D}$, and $\boldsymbol{W} \boldsymbol{S} \boldsymbol{M}$ if and only if $\varphi \in \mathcal{H}$.

(c) $\boldsymbol{N G}, \boldsymbol{C D D}$, and $\boldsymbol{S M}$ if and only if $\varphi \in \mathcal{R}$.

(d) $\boldsymbol{M D}$, and $\boldsymbol{S} \boldsymbol{M}$ if and only if $\varphi \in \mathcal{S}$.

(d) $\boldsymbol{M D}$, and $\boldsymbol{S S M}$ if and only if $\varphi \in \mathcal{P} \mathcal{S}$.

Certainly, the axiomatizations (d) and (e) in Theorem 3.10 are closely connected with the corresponding ones in Nowak and Radzik (1995). However, it seems that in the literature so far it has not been recognized that $\mathbf{S M}$ can replace $\mathbf{M}$ and $\mathbf{M o}$ in this axiomatization. Mo is not implied by SM. On the contrary, SM can be considered weaker than $\mathbf{M}$ and $\mathbf{M o}$ together as our next result shows: if a TU-value $\varphi$ meets $\mathbf{E}, \mathbf{M}, \mathbf{M o}$, and $\mathbf{C D D}, \varphi$ is a random order value while a TU-value that meets $\mathbf{E}, \mathbf{S M}$, and $\mathbf{C D D}$ does not have to be (see Remark 5.5). The following corollary is related to Theorem 3.2. $\mathbf{M}$ and $\mathbf{C D D}$ (and NG in case (a)) replace $\mathbf{N}$ and $\mathbf{A}$.

Corollary 3.8. A TU-value $\varphi$ satisfies $\boldsymbol{E}, \boldsymbol{M}, \boldsymbol{C D D}$, and 
(a) $\boldsymbol{N G}$ if and only if $\varphi \in \mathcal{M}$.

(b) $\boldsymbol{P o}$ if and only if $\varphi \in \mathcal{H}$.

(c) Mo if and only if $\varphi \in \mathcal{R}$.

(d) $\boldsymbol{S S S}$ if and only if $\varphi \in \mathcal{S}$.

(e) $\boldsymbol{W S S}$ if and only if $\varphi \in \mathcal{P} \mathcal{S}$.

\section{Conclusion}

Additivity does not really have great economic significance. Casajus and Huettner (2014) are of the opinion that additivity "is a rather technical condition with little economic content." In contrast, monotonicity properties appear to be more practical and the payoffs achieved more comprehensible. Of course, coalitional differential dependence has a small aftertaste of weightedness. However, while the payoff ratios of dependent players by mutual dependence are directly fixed by the payoff ratios in unanimity games, this is not the case by coalitional differential dependence. Parallel axiomatization has many advantages, in particular when the different TU-value classes are in a proper subset interrelationship. E. g., the proofing can be much shorter by using a complete proof only for the largest superclass. For the subclasses, we may only need proofs for the change steps or we may refer to known older results. If the TU-value classes are in a proper subset interrelationship, we can also derive new results that are not explicitly specified in the parallel axiomatization. For example, we can directly see in our results that a multiweighted Shapley value that satisfies weakly strong monotonicity is a Harsanyi payoff or a weighted Shapley value that satisfies strictly strong monotonicity is a positively weighted Shapley value.

\section{Appendix}

\subsection{A remark and basic lemmas, used in the proofs}

Remark 5.1. It is well-known that (4) and (5) can be replaced equivalently by

$$
\Delta_{v}(S)=\left\{\begin{array}{l}
\Delta_{w}(T)+c, \text { if } S=T \\
\Delta_{w}(S), \text { otherwise. }
\end{array}\right.
$$

Lemma 5.2. (Casajus and Huettner, 2008). If $i \in N$ and $v, w \in \mathbb{V}$, then $M C_{i}^{v}(S)=$ $M C_{i}^{w}(S)$ for all $S \subseteq N \backslash\{i\}$ if and only if $\Delta_{v}(S \cup\{i\})=\Delta_{w}(S \cup\{i\})$ for all $S \subseteq N \backslash\{i\}$.

Lemma 5.3. If $i \in N$ and $v, w \in \mathbb{V}$, then $M C_{i}^{v}(T)>M C_{i}^{w}(T), T \subseteq N \backslash\{i\}$, and $M C_{i}^{v}(S)=$ $M C_{i}^{w}(S)$ for all $S \subseteq N \backslash\{i\}, S \neq T$, if and only if $\Delta_{v}(S \cup\{i\})=\Delta_{w}(S \cup\{i\})$ and $\Delta_{v}(T \cup\{i\})>$ $\Delta_{w}(T \cup\{i\})$.

Proof. $\Rightarrow$ : Let $M C_{i}^{v}(T)>M C_{i}^{w}(T), T \subseteq N \backslash\{i\}$, and $M C_{i}^{v}(S)=M C_{i}^{w}(S)$ for all $S \subseteq$ $N \backslash\{i\}, S \neq T$. We have

$$
M C_{i}^{v}(T)=\sum_{S \subseteq T} \Delta_{v}(S \cup\{i\})>\sum_{S \subseteq T} \Delta_{w}(S \cup\{i\})=M C_{i}^{w}(T)
$$


and it follows, by Lemma 5.2, $\Delta_{v}(T \cup\{i\})>\Delta_{w}(T \cup\{i\})$.

$\Leftarrow$ : Let $\Delta_{v}(S \cup\{i\})=\Delta_{w}(S \cup\{i\})$ for all $S \subseteq N \backslash\{i\}, S \neq T$, and $\Delta_{v}(T \cup\{i\})>\Delta_{w}(T \cup$ $\{i\}), T \subseteq N \backslash\{i\}$. We have, by Lemma 5.2,

$$
M C_{i}^{v}(T)=\sum_{S \subseteq T} \Delta_{v}(S \cup\{i\})>\sum_{S \subseteq T} \Delta_{w}(S \cup\{i\})=M C_{i}^{w}(T) .
$$

Lemma 5.4. If a TU-value $\varphi$ satisfies $\boldsymbol{E}$ and $\boldsymbol{M D}$, then $\varphi$ also satisfies $\boldsymbol{N G}$.

Proof. Let $\varphi$ satisfy $\mathbf{E}$ and $\mathbf{M D}$. We show, by contradiction, that $\varphi$ meets NG: If $\varphi$ does not satisfy $\mathbf{N G}$, we have in a null game at least one $i \in N$ such that $\varphi_{i}(\mathbf{0}) \neq 0$. If $N=\{i\}$ we immediately have a contradiction due to $\mathbf{E}$, if $|N| \geq 2$, the contradiction follows due to $\mathbf{M D}$ and $\mathbf{E}$, because we have

$$
\sum_{j \in N} \varphi_{j}\left(u_{N}\right)=\frac{\sum_{j \in N} \varphi_{j}(\mathbf{0})}{\varphi_{i}(\mathbf{0})} \varphi_{i}\left(u_{N}\right) \Leftrightarrow 1=0 . \not
$$

\subsection{Proofs}

\subsubsection{Proof of Proposition 3.5}

$\Rightarrow$ : Let $\varphi$ be a TU-value that satisfies WSM and let the preconditions be as in CSM. Note that $\varphi$ satisfies also $\mathbf{M}$ and thus, by Proposition 3.4, CSE. Therefore, we only have to show that $\varphi_{j}(v) \geq \varphi_{j}(w)$ for all $j \in T$.

By (5) and Remark 5.1, we have $\Delta_{v}(S)=\Delta_{w}(S)$ for all $S \neq T$ and $\Delta_{v}(T)>\Delta_{w}(T)$. By Lemma 5.3, we have, $M C_{j}^{v}(T \backslash\{j\})>M C_{j}^{w}(T \backslash\{j\}), T \ni j$, and $M C_{j}^{v}(S \backslash\{j\})=$ $M C_{j}^{w}(S \backslash\{j\})$ for all $S \ni j, S \neq T$, and the claim follows by WSM.

$\Leftarrow$ : Let $\varphi$ be a TU-value that satisfies CSM and let the preconditions be as in WSM. Note that $\varphi$ satisfies also CSE and thus, by Proposition 3.4, M. Therefore, we only have to show that $\varphi_{i}(v) \geq \varphi_{i}(w)$ for all $i \in N$ such that $M C_{i}^{v}(T)>M C_{i}^{w}(T)$ for a $T \subseteq N \backslash\{i\}$ and $M C_{i}^{v}(S)=M C_{i}^{w}(S)$ for all $S \subseteq N \backslash\{i\}, S \neq T$.

By Lemma 5.3, we have $\Delta_{v}(S \cup\{i\})=\Delta_{w}(S \cup\{i\})$ and $\Delta_{v}(T \cup\{i\})>\Delta_{w}(T \cup\{i\})$. Thus, by Remark 5.1, the preconditions in CSM are satisfied and the claim follows by CSM.

\subsubsection{Proof of Theorem 3.7}

$\Rightarrow$ : It is well-known that all TU-values $\varphi \in \mathcal{M}$ satisfiy $\mathbf{E}$ and, by (3), it is obvious that NG is satisfied too. By Lemma 5.2, Remark 5.1, and (3), CSE is satisfied and therefore, by Proposition 3.4, $\mathrm{M}$ too.

We show CDD. Let $\alpha, \beta \in \mathbb{R}, v, w \in \mathbb{V}, \lambda^{+} \in \Lambda^{+} N, S \subseteq N$, and $i, j \in S$ be dependent in $v$ and $w$. By (1) and (3), we have for $k \in\{i, j\}$

$$
M_{k}^{\lambda_{-}^{+}}\left(v+\alpha \cdot u_{S}\right)-M_{k}^{\lambda_{-}^{+}}(v)=\lambda_{S, k}^{+} \cdot \alpha \text { and } M_{k}^{\lambda_{-}^{+}}\left(w+\beta \cdot u_{S}\right)-M_{k}^{\lambda_{-}^{+}}(w)=\lambda_{S, k}^{+} \cdot \beta
$$


and CDD is satisfied. Thus, existence is shown for the (a) part. By Remark 3.1, $\mathcal{H}$ and $\mathcal{R}$ are subsets of $\mathcal{M}$, by Dragan (1992, Theorem 4.8), the TU-values from $\mathcal{R}$ satisfy $\mathbf{S M}$, and, by Nowak and Radzik (1995, Theorem 2.4 and Remark 2.3) the TU-values from $\mathcal{S}$ and $\mathcal{P} \mathcal{S}$ satisfy MD. The only thing that remains to be shown in the existence part is that in the cases (b), (d), and (e) the TU values of the corresponding classes meet the related monotonicity properties:

(b) By Lemma 5.3, Remark 5.1, and (2), CSM is satisfied and therefore, by Proposition 3.5, WSM too.

(d)/(e) By Nowak and Radzik (1995, Theorem 2.1, Equation (2.2)), it is immediate that all $\varphi \in \mathcal{S}$ satisfy $\mathbf{S M}$ and all $\varphi \in \mathcal{P} \mathcal{S}$ satisfy $\mathbf{S S M}$ (take $\sigma=\{N\}$ ).

$\Leftarrow$ : (a) $)^{3}$ Let $v \in \mathbb{V}$ and $\varphi$ be a TU-value that satisfies $\mathbf{E}, \mathbf{N G}, \mathbf{C D D}$, and $\mathbf{M}$ and therefore, by Lemma 5.2, CSE. We show, by induction on the size $r:=\mid\{R \subseteq N: R$ is essential in $v\} \mid$, that $\varphi \in \mathcal{M}$.

Initialization: Let $r=0$. By NG, we have $\varphi \in \mathcal{M}$.

Induction step: Assume that $\varphi \in \mathcal{M}$ holds to $\varphi$ if $r \geq 0, r$ arbitrary $(I H)$. Now let precisely $r+1$ coalitions $K_{\ell} \subseteq N, 1 \leq \ell \leq r+1$, be essential in $v$. We define $K$ as the intersection of all $K_{\ell} \subseteq N, 1 \leq \ell \leq r+1$ :

$$
K:=\bigcap_{1 \leq \ell \leq r+1} K_{\ell}
$$

We are dealing with two different cases: (i) $i \in N \backslash K$ and (ii) $i \in K$.

(i) Each $i \in N \backslash K$ is contained in at most $r$ essential coalitions $K_{\ell}$ in $v$ and we have at least one essential coalition $R_{i}$ in $v$ such that $i \notin R_{i}$. Therefore, a $v_{i} \in \mathbb{V}$ exists for all $i \in N \backslash K$ such that $\Delta_{v_{i}}\left(R_{i}\right)=0, \Delta_{v_{i}}(S)=\Delta_{v}(S)$ for all $S \subseteq N, S \neq R_{i}$, and $\Delta_{v}\left(R_{i}\right)=\Delta_{v_{i}}\left(R_{i}\right)+c_{i}, c_{i} \in$ $\mathbb{R}, c_{i} \neq 0$. It follows, by Remark 5.1 and CSE, $\varphi_{i}(v)=\varphi_{i}\left(v_{i}\right)$ for all $i \in N \backslash K$ and thus, by $(I H)$,

$$
\varphi_{i}(v)=M_{i}^{\lambda_{-}^{+}}(v) \text { for some } \lambda^{+} \in \Lambda^{{ }^{+}} N \text { and all } i \in N \backslash K \text {. }
$$

(ii) If $K=\{i\}$, we have, by $\mathbf{E}$ and (i), $\varphi_{i}(v)=M_{i}^{\lambda_{-}^{+}}(v)$ for some $\lambda^{+} \in \Lambda^{+} N$. If $|K| \geq 2$, by (1), all $j \in K$ are dependent in $v$, in $u_{K}$, and in $\mathbf{0}$. We have, by $\mathbf{N G}, \varphi_{i}\left(\mathbf{0}+u_{K}\right)-\varphi_{i}(\mathbf{0})=$ $\varphi_{i}\left(u_{K}\right)$ for all $i \in K$ and, by $\mathbf{E}, \sum_{i \in K} \varphi_{i}\left(u_{K}\right)=1$. Thus, at least one $j \in N$ exists such that $\varphi_{j}\left(u_{K}\right) \neq 0$. Let $v^{\prime} \in \mathbb{V}$ such that $\Delta_{v^{\prime}}(K)=0, \Delta_{v^{\prime}}(S)=\Delta_{v}(S)$ for all $S \subseteq N, S \neq K$, and therefore, by $(I H), \varphi_{i}\left(v^{\prime}\right)=M_{i}^{\lambda_{-}^{+}}\left(v^{\prime}\right)$ for some $\lambda^{+} \in \Lambda^{+}{ }^{+}$and all $i \in K$. It follows for all $j \in K$ with $\varphi_{j}\left(u_{K}\right) \neq 0$ :

$$
\begin{aligned}
\sum_{i \in K}\left[\varphi_{i}(v)-\varphi_{i}\left(v^{\prime}\right)\right] \underset{(\mathbf{C D D})}{=} \sum_{i \in K}\left[\frac{\varphi_{i}\left(u_{K}\right)}{\varphi_{j}\left(u_{K}\right)}\left[\varphi_{j}(v)-\varphi_{j}\left(v^{\prime}\right)\right]\right] \\
\underset{(\mathbf{E})}{=} v(N)-v^{\prime}(N)-\sum_{i \in N \backslash K}\left[M_{i}^{\lambda_{-}^{+}}(v)-M_{i}^{\lambda_{-}^{+}}\left(v^{\prime}\right)\right]=\Delta_{v}(K) \\
\Rightarrow \varphi_{j}(v)=\varphi_{j}\left(u_{K}\right) \Delta_{v}(K)+\varphi_{j}\left(v^{\prime}\right)=M_{j}^{\lambda_{-}^{+}}\left(v^{\prime}\right)+c_{j} \Delta_{v}(K) .
\end{aligned}
$$

Additionally, by CDD, we have $\varphi_{i}(v)=\varphi_{i}\left(v^{\prime}\right)=M_{i}^{\lambda_{-}^{+}}\left(v^{\prime}\right)$ for all $i \in K$ with $\varphi_{i}\left(u_{K}\right)=0$. In total, we get $\varphi_{i}(v)=M_{i}^{\lambda^{\prime+}}(v)$ for all $i \in K$ and a $\lambda^{\prime^{+}} \in \Lambda^{{ }^{+}}{ }^{N}$.

\footnotetext{
${ }^{3}$ The proof follows in some parts the uniqueness part of the proof of Theorem 2 in Besner (2019).
} 
(b) The proof is completely analogues to the proof of case (a).

(c) $\varphi$ satisfies $\mathbf{S M}$ and therefore $\mathbf{M}$. Thus, $\varphi$ is a multiweighted Shapley value and the claim follows by Dragan (1992, Theorem 4.8).

(d) MD implies CDD. Thus $\varphi$ satisfies E, CDD, M, and, by Lemma 5.4, NG. Therefore we have $\varphi \in \mathcal{M}$. By Dragan (1992, Proposition 4.7), $\varphi$ satisfies Mo and hence, by Nowak and Radzik (1995, Theorem 2.4), we obtain $\varphi \in \mathcal{S}$.

(e) By Lemma 5.4, $\varphi$ satisfies NG. Therefore, by E, NG, and SSM, we have $\varphi_{i}\left(u_{N}\right)>0$ for all $i \in N$. Let $v \in \mathbb{V}$ and $i, j \in N$ be dependent in $v$. By MD, we have

$$
\frac{\varphi_{i}(v)}{\varphi_{i}\left(u_{N}\right)}=\frac{\varphi_{j}(v)}{\varphi_{j}\left(u_{N}\right)}
$$

and axiom A4 ( $\omega$-Mutual Dependence) in Nowak and Radzik (1995) is satisfied for the special case $\sigma=\{N\}$. By Remark 3.3 and case (a), we have $\varphi \in \mathcal{M}$. Therefore, $\varphi$ satisfies also linearity and the null player property and, by Nowak and Radzik (1995, Theorem 2.1 with $\sigma=\{N\})$, we get $\varphi \in \mathcal{P S}$.

\subsubsection{Proof of Corollary 3.8}

According to the Theorems 3.2 and 3.7 , and due to the property that $\mathcal{H}, \mathcal{R}, \mathcal{S}$, and $\mathcal{P} \mathcal{S}$ are all subsets of $\mathcal{M}$, we only have to show uniqueness.

(a) The result is already shown in Theorem 3.7 case (a).

(b)/(c)/(d)/(e) Let now $\varphi^{\mathcal{H}}, \varphi^{\mathcal{R}}, \varphi^{\mathcal{S}}$, and $\varphi^{\mathcal{P} \mathcal{S}}$ be TU-values that satisfy all mentioned properties in the related cases. If $|N|=1, \mathbf{N G}$ is implied by $\mathbf{E}$. Let now $|N| \geq 2$. Obviously, Po, Mo, and WSS each imply, together with E, NG. Also SSS together with E imply NG, which we will show by contradiction. Assume $\varphi$ does not meet NG. By E, we have at least one $i \in N$ with $\varphi_{i}(\mathbf{0})>0$. It follows, by $\mathbf{S S S}$, that we have $\varphi_{j} \geq 0$ for all $j \in N \backslash\{i\}$ which contradicts E. Therefore, all TU-values of all classes must be multiweighted Shapley values and the claim follows by Theorem 3.2.

\subsection{Logical independence}

Remark 5.5. Let $v \in \mathbb{V}$. The axioms in Theorem 3.7 are logically independent:

- E: The TU-value $\varphi:=2 S h$ satisfies all axioms but $\mathbf{E}$.

- NG: Let $N=\{i, j\}$. The TU-value $\varphi$, defined by $\varphi_{i}(v):=S h_{i}(v)+1, \varphi_{j}(v):=S h_{j}(v)-1$, satisfies E, M, WSM, SM, and CDD, but not NG.

- CDD/MD: Let $N=\{i, j\}$. The TU-value $\phi$, defined by (6)/(7), satisfies all axioms but CDD and MD.

- M/WSM/SM/SSM: The TU-value $\varphi:=\frac{v(N)}{|N|}$ satisfies E, NG, CDD, and MD, but not M, WSM, SM, and SSM.

Remark 5.6. Let $v \in \mathbb{V}$. The axioms in Corollary 3.8 are logically independent:

- E: The TU-value $\varphi:=2 S h$ satisfies all axioms but E. 
- M: The TU-value $\varphi:=\frac{v(N)}{|N|}$ satisfies all axioms but $\mathbf{M}$.

- CDD: Let $N=\{i, j\}$. The TU-value $\phi$, defined by (6)/(7), satisfies all axioms but CDD.

- NG: Let $N=\{i, j\}$. The TU-value $\varphi$, defined by $\varphi_{i}(v):=S h_{i}(v)+1, \varphi_{j}(v):=S h_{j}(v)-1$, satisfies $\mathbf{E}, \mathbf{M}$, and $\mathbf{C D D}$, but not $\mathbf{N G}$.

- Po/Mo/SSS/WSS: Let $N=\{i, j\}$. The multiweighted Shapley value $\varphi$, defined by $\varphi_{i}(v):=v(\{i\})+2 \Delta_{v}(\{i, j\})$ and $\varphi_{j}(v):=v(\{j\})-\Delta_{v}(\{i, j\})$ satisfies E, M, and CDD, but not Po, Mo, SSS, and WSS.

\section{References}

Besner, M. (2019). Axiomatizations of the proportional Shapley value. Theory and Decision https://doi.org/10.1007/s11238-019-09687-7

van den Brink, R., Funaki, Y., \& Ju, Y. (2013). Reconciling marginalism with egalitarianism: consistency, monotonicity, and implementation of egalitarian Shapley values. Social Choice and Welfare, 40(3), 693714.

Casajus, A., \& Huettner, F. (2008) Marginality is equivalent to coalitional strategic equivalence. Working paper.

Casajus, A., \& Huettner, F. (2014) Weakly monotonic solutions for cooperative games. Journal of Economic Theory, 154, 162-172.

Casajus, A. (2019). Relaxations of symmetry and the weighted Shapley values. Economics Letters, 176, 7578.

Chun, Y. (1989) A new axiomatization of the Shapley value. Games and Economic Behavior 1(2), 119-130.

Derks, J., Haller, H., \& Peters, H. (2000). The selectope for cooperative games. International Journal of Game Theory, 29(1), 23-38.

Dragan, I. C. (1992) Multiweighted Shapley values and random order values. University of Texas at Arlington.

Hammer, P. L., Peled, U. N., \& Sorensen, S. (1977). Pseudo-boolean functions and game theory. I. Core elements and Shapley value. Cahiers du CERO, 19, 159-176.

Harsanyi, J. C. (1959). A bargaining model for cooperative n-person games. In: A. W. Tucker \& R. D. Luce (Eds.), Contributions to the theory of games IV (325-355). Princeton NJ: Princeton University Press.

Joosten, R. A. M. G. (1996). Dynamics, equilibria, and values. (Doctoral dissertation, Maastricht University).

Kalai, E., \& Samet, D. (1987) On weighted Shapley values. International Journal of Game Theory 16(3), 205-222.

Nowak, A. S., \& Radzik, T. (1995). On axiomatizations of the weighted Shapley values. Games and Economic Behavior, 8(2), 389-405.

Shapley, L. S. (1953a). Additive and non-additive set functions. Princeton University.

Shapley, L. S. (1953b). A value for n-person games. H. W. Kuhn/A. W. Tucker (eds.), Contributions to the Theory of Games, Vol. 2, Princeton University Press, Princeton, 307-317.

Vasil'ev, V. A. (1975). The Shapley value for cooperative games of bounded polynomial variation. Optimizacija Vyp, 17, 5-27.

Vasil'ev, V. A. (1978). Support function of the core of a convex game. Optimizacija Vyp, 21, 30-35.

Vasil'ev, V. A. (1981). On a class of imputations in cooperative games, Soviet Mathematics Dokladi 23 53-57.

Weber, R. J. (1988). Probabilistic values for games. In A.E. Roth (Ed.), The Shapley value, essays in honor of L.S. Shapley (pp. 101-119). Cambridge: Cambridge University Press.

Young, H. P. (1985). Monotonic solutions of Cooperative Games. International Journal of Game Theory, $14(2), 65-72$. 